\title{
Metas inflacionárias: a análise convencional e um modelo alternativo
}

\author{
LUIZ ANTONIO DE OLIVEIRA LIMA*
}

Inflation targeting: the conventional analysis and an alternative model. This article has two aims: the first one is to present a formal model of the monetary policy identified generally as "inflation targeting policy", an instrument of intervention of the central bank, through the short run nominal interest rate. The second aim is to discuss and criticize the theoretical assumptions of the model specially the concepts of "natural rate of interest" and of potential product presented by the "augmented Philips curve"; and to present a more realistic control of inflation targeting which does not assume the hypotheses above, and in which inflation targeting is based on the control of real rate of interest.

Keywords: Short-run interest rate; inflationary targeting, potential output; nominal monetary rules, natural interest rate.

JEL Classification: E00; E4; E52; E58.

A adoção de um sistema de "metas inflacionárias", ou seja, uma forma de regra baseada em resultados, tem caracterizado a política monetária de alguns dos principais bancos centrais das economias capitalistas. De modo geral, podese definir um sistema de metas inflacionárias como uma regra para ajustar os instrumentos de política diante de desvios de determinadas variáveis, no caso específico diante de oscilações inflacionárias. Segundo a interpretação corrente, o alegado sucesso de tal política pode ser explicado pela utilização de um modelo com uma fundamentação teórica diferente da regra monetarista, que tinha como meta o controle dos agregados monetários, sem definir explicitamente os resultados a serem alcançados em termos de taxas inflacionárias. Tal sistema, ligado ao

\footnotetext{
* Professor da Escola de Administração de Empresas de São Paulo da Fundação Getulio Vargas FGV/EAESP. Este artigo é parte de projetos financiados pela GV/Pesquisa.O autor agradece sugestões e críticas de Robert Nicol e de dois referees anônimos. Submetido: Fevereiro 2006; Aceito: Outubro 2006.
} 
nome de Milton Friedman, baseava-se na hipótese de que embora os agentes privados não tivessem condições de informação para estabelecer resultados ótimos, a economia, através da competição entre esses agentes poderia fazê-lo, de tal maneira que aos bancos centrais competia apenas criar a disponibilidade monetária compatível com o crescimento potencial da economia.

O argumento para a adoção de metas inflacionárias, ao contrário da suposição monetarista, admite que tanto o banco central quanto os agentes privados sabem como a economia funciona, e em função disso formam suas expectativas racionalmente. Nesta situação, as pessoas passam a entender o comportamento do banco central de tal modo que se este tomar decisões incompatíveis com os objetivos do público, ele errará sistematicamente, geralmente em direção de uma inflação excessiva. O estabelecimento por parte das autoridades monetárias de metas, por exemplo, uma meta de inflação compatível com as condições da economia, consistiria na melhor forma de manter uma taxa de inflação estável e baixa e a economia sem turbulência. De acordo com John Taylor (1998), “correntemente diversos bancos centrais parecem estar seguindo políticas que implicam aproximadamente uma meta de $2 \%$ ao ano. O Banco da Inglaterra, o Banco da França, o Bundesbank estão objetivando tal meta inflacionária" (p. 46), chegando mesmo a afirmar que, no período de 1987 a 1995, o Federal Reserve praticou uma política que mesmo não explícita pode ser interpretada como um regime de metas, pois os juros praticados por essa instituição podem ser explicados com precisão pela regra inflacionária por ele proposta.

Devemos, no entanto, ser cautelosos em relação a tal informação. De acordo com o ex-presidente do Federal Reserve, Allan Greenspan, (2004) “embora as regras captem algumas configurações econômicas, em suas linhas gerais, daquilo que têm sido feito nos últimos quinze anos, como tem sido sugerido por seus proponentes, em pontos cruciais, como aqueles de nossa história econômica recente (o crash do mercado de ações de 1987, as crises de 1997 e 1998, e os eventos que se seguiram em setembro de 2001) regras simples serão inadequadas quer como descrição ou prescrição de políticas; a política do FED baseada em um paradigma de administração de riscos tem dependido de previsões de probabilidades desenvolvidas a partir de grandes modelos macroeconômicos, numerosos sub-modelos e julgamentos menos precisos matematicamente. Tais julgamentos por sua natureza são baseados em situações históricas particulares que não podem ser associadas a uma simples análise de variância” (p. 39).

Greenspan observa ainda que não há evidência de que um simples anúncio que um banco central procura se engajar em um sistema de metas inflacionárias aumente sua credibilidade, ou seja, a certeza de que o Banco Central procurará manter a estabilidade dos preços. Na verdade, "não saberemos, seguramente, o significado do regime de metas inflacionárias até que a economia mundial seja submetida a choques de suficiente grandeza para aferir a performance diferencial dos países que não empregam formalmente o anúncio de metas inflacionárias. Até agora, a inflação tem caído para aqueles países que têm metas inflacionárias como também para aqueles que não têm” (p. 40). 
A partir das considerações acima podemos definir os objetivos deste trabalho:

a) especificar os conceitos convencionais de regras e metas inflacionárias e, a partir de tais conceitos, discutir uma regra específica, ou seja, a de metas inflacionárias;

b) dada a observação de Allan Greenspan de que seria prematuro um estudo comparativo da performance de economias com decisões discricionárias e outras baseadas em sistema de regras, se procurará fazer uma avaliação crítica deste último, tomando-se como base a coerência teórica dos conceitos que o fundamentam, ou seja: a taxa de juro de equilíbrio de longo prazo, que no caso deverá corresponder à taxa de juro natural de Wicksell; e o nível de renda real de pleno emprego, o qual teria como correlato a taxa de desemprego não aceleradora de inflação (NAIRU), bem como sugerir as linhas gerais que norteiam uma política monetária realista.

\section{A FUNÇÃO DE REAÇÃO, DEMANDA AGREGADA E METAS INFLACIONÁRIAS}

Temos uma função de reação toda vez que as autoridades monetárias definem quantitativamente qual sua reação (variação da taxa de juro) a variações efetivas nas taxas de inflação e/ou no hiato do produto ou na taxa de desemprego, tendo em vista uma determinada meta de inflação ou crescimento.

Uma "função de reação" pode ter a seguinte expressão

$$
i_{r}=h_{0}+h_{1} \dot{p}
$$

sendo $i_{\mathrm{r}}$ taxa de juro real, $h_{1}$ o parâmetro que reflete a sensibilidade do Banco central a variações na taxa de inflação.

A função linear acima pode ser representada com inclinação sempre positiva $h_{1}$ e com intercepto $h_{0}$, de tal maneira que quanto maior for $h_{0}$, isto é, mais rigorosos os objetivos inflacionários, mais uma elevação de taxa de inflação efetiva, dado $h_{1}$, mais alta será a taxa de juro real a ser atingida. Para tanto, segundo Taylor (2000), o "Banco Central deve tomar decisões que elevem" a taxa de juro de curto prazo (no caso americano a taxa dos fundos federais) o suficiente para elevar a taxa de juro real para o valor adequado" (p. 91).

Sendo a taxa de juro real definida a partir de

$$
i_{r}=i-p
$$

sendo $i$ a taxa nominal de juro e $\dot{p}$ a taxa de inflação esperada.

As implicações da função (1.1) ficarão claras se a combinarmos com uma função $I S$, dada pela equação abaixo

$$
Y=\alpha_{0}-\alpha_{1} i_{r}
$$

na qual $a_{0}$ corresponde ao valor do gasto autônomo da economia e o coefi- 
ciente $a_{1}$ indica as variações dos gastos sensíveis a taxa de juro, $i_{r}$, e $Y$ a renda real (uma redução de $i_{r}$ implicará uma elevação de $Y$ ).

Assim, substituindo-se o valor de $i_{r}$ dado em (1.1), na equação (1.3), obtemos a equação, ou

$$
\begin{gathered}
Y=a_{0}-a_{1}\left(h_{0}+h_{1} \dot{p}\right), \\
\text { ou } \\
Y=a_{0}-a_{1} h_{0}-a_{1} h_{1} \dot{p}
\end{gathered}
$$

A equação (1.4), que pode ser identificada como uma função de Demanda Agregada $(D A)$, estabelece uma relação inversa entre taxa de inflação $(\dot{p})$ e renda $(Y)$ de tal maneira que para valores elevados de $\dot{p}$ temos valores reduzidos de $Y$ e vice-versa. Tal função está representada no Gráfico 1 por uma linha de inclinação negativa de tal modo que se pode interpretar movimentos ao longo dela como conseqüência de mudanças na taxa de inflação, levando a alterações no produto. De outro lado deslocamentos em $D A$ decorrem de alterações no valor dos gastos autônomos dados por $a_{0}$

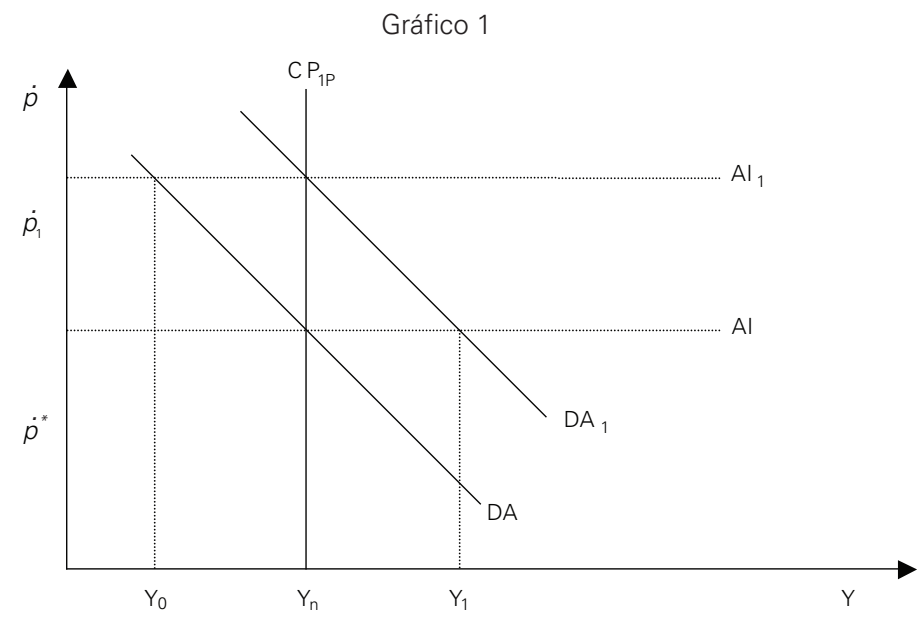

Uma das implicações da análise acima é de que a taxa de juro real de equilíbrio $i_{r}^{*}$, isto é, a taxa de juro correspondente a um valor do produto de pleno emprego ou potencial $Y_{n}$, independe totalmente da política monetária, sendo determinada apenas pelo gasto autônomo da economia dado na função IS. Tal pode ser evidenciado a partir da equação (1.3) na qual se substitui $Y$, por $Y$ potencial, $Y_{n}$, e $i_{r}$ por $i_{r}^{*}$, assim

$$
Y_{n}=a_{0}-a_{1} i_{r}^{*}
$$

ou

$i_{r}^{*}=\frac{a_{0}-Y_{n}}{a_{1}}$ 
De forma análoga, podemos determinar uma inflação correspondente ao equilíbrio de longo prazo, substituindo-se na função de reação do Banco Central os valores correspondentes a $i_{r}$ e $Y$ por $i_{r}^{*}$ e $Y_{n}$

$$
\dot{p}^{*}=\frac{i_{r}^{*}-h_{0}}{h_{1}}
$$

\section{Política monetária e inflação}

A realização de uma política de metas inflacionárias requer não só a explicitação de uma taxa de juro de equilíbrio de longo prazo, $i_{r}^{*}$, como também a determinação de um nível de renda potencial ou pleno emprego $Y_{n}$. A introdução desta variável no modelo requer que se estabeleça uma hipótese sobre como a inflação se relaciona com a renda: a hipótese a ser considerada é a da "curva de Philips aumentada pelas expectativas", segundo a qual ocorrem mudanças na taxa de inflação toda vez que a renda efetiva esteja acima ou abaixo da renda de pleno emprego $\left(Y_{n}\right)$. A fórmula de tal função é dada por

$$
p=p_{-1}+c \frac{\left(Y-Y_{n}\right)}{Y_{n}}
$$

Assim, a inflação se elevará quando o "lag" $\left(Y-Y_{n}\right)$ for maior do que zero, e cairá quando for menor, de tal modo que quando $Y=Y_{n}, p=p_{-1}$. A função (1.7) pode ser representada no Gráfico 1 como uma vertical ao eixo horizontal, traçada a partir da renda de pleno emprego $Y_{n}$ e que será identificada por $\mathrm{CP}_{1 p}$. Considere que em tal gráfico $\mathrm{CP}_{1 p}$ e $D A$ estejam determinando uma taxa de inflação que pode ser considerada adequada, ou seja, $\dot{p}=\dot{p}^{*}$, mas que uma elevação em $a_{o}$, (gasto autônomo), desloque $D A$ para $D A_{1}$ (ver equação 1.4 ), fazendo com que $Y_{n}$ se eleve para $Y_{1}$. Como na nova situação a economia está produzindo além de sua capacidade potencial, os preços começam a se elevar, ao longo $D A_{1}$, de tal modo que $\ddot{p} "$ se altera para $\dot{p}_{1}$, o que é mostrado pelo deslocamento da linha de ajuste inflacionário de $A I$ para $A I_{1}$. Neste caso, de acordo com a equação 1.5, haverá uma elevação da taxa de juro real decorrente do aumento do gasto autônomo $\left(a_{0}\right)$. No entanto, se $\ddot{p}^{*}$ continuar sendo a meta inflacionária ela poderá ser atingida desde que o banco central eleve o valor do parâmetro $h_{0}$, em sua "função de reação", determinando um aumento em $i_{r}$. Tal decisão pode ser refletida no Gráfico 1 mediante um deslocamento de $D A_{1}$ para a esquerda, (ver equação 1.4) digamos para a antiga posição de $D A$ levando à renda $Y_{0}$. Nesta situação, de acordo com a hipótese expressa pela equação (1.7) os preços cairiam até que $\dot{p}$ voltasse para o nível $\ddot{p}^{*}$. De acordo com a equação (1.5), no entanto, a taxa de juro real de equilíbrio $\left(i_{r}^{*}\right)$ se estabelecerá em um valor mais elevado, em função do aumento da participação do gasto autônomo na demanda agregada, isto é, pelo aumento de $a_{0}$. Além disso, se $i_{r}^{*}$ se eleva e a taxa de inflação retorna a $\ddot{p}^{*}$ 'o 
banco central deverá estar praticando uma taxa nominal de juro mais alta do que na situação em que $a_{\mathrm{o}}$ não tinha se elevado.

Em resumo, a análise acima restringe a função da política monetária à determinação apenas de uma meta inflacionária através do controle de $D A$, não devendo ter nenhum papel ativo na determinação do nível ótimo de produção, que será alcançado sempre a longo prazo, independente da política monetária. Esta hipótese reflete a superneutralidade da moeda. De maneira mais específica, mesmo que em situação de desemprego seja utilizado algum estímulo para abreviar tal ajuste, de acordo com o modelo acima, não compete à política econômica estabelecer uma meta de produção, a qual é determinada pelo lado da oferta da economia através da curva de Philips ampliada pelas expectativas.

\section{A determinação da meta inflacionária}

O modelo exposto anteriormente explicita a lógica e os pressupostos da determinação da variável instrumental, taxa de juro, que permite chegar a uma meta inflacionária. Falta, no entanto, estabelecer a forma pela qual se determina a meta inflacionária. O método geral é a minimização de uma função de perda do banco central, na qual se supõe que variações da inflação em relação a uma meta inflacionária e variações do produto em relação ao produto de pleno emprego implicam uma perda para o banco central, a qual deverá ser reduzida a um valor mínimo pela escolha de uma taxa inflacionária específica que será alcançada pela taxa de juro a ser praticada pelo banco central ${ }^{1}$.

Uma forma alternativa de determinação da variável instrumental, taxa de juro, decorre diretamente de uma regra. A mais famosa dessas regras foi formulada por Taylor (1993), utilizada, entre outros, pelos bancos centrais da Inglaterra, Nova Zelândia, Canadá e Brasil (para o caso brasileiro ver Minella et al., 2002, p. 11). Sua formulação algébrica é dada por

$$
i=\dot{p}+i_{r}^{*}+h_{i}\left[\dot{p}-\dot{p}^{*}\right]+h_{2} y
$$

onde ${ }^{y=100\left(\frac{Y-Y_{n}}{Y_{n}}\right)}$ e Y é o PIB real e $Y_{n}$ é o PIB tendencial. No exemplo fornecido por Taylor, este atribui valor igual a 0.5 para $h_{1}$ e $h_{2}$, bem como supõe que $\ddot{p}^{*}=2 \%$ e $i_{r}^{*}=2 \%$ (assim como admite que a taxa de crescimento tendencial da economia americana foi de 2,2\% entre $1984-91$ a 1992-93). Com tais suposições, a fórmula acima (1.8) se reduz a

$$
i=\dot{p}+2+0,5(\dot{p}-2)+0,5 y
$$

\footnotetext{
${ }^{1}$ Para um exemplo bastante claro de tal procedimento, ver Mankiw (1998), pp. 294-5.
} 
Para uma taxa de inflação de $2 \%$ e um crescimento real da economia de $2,2 \%$ (igual à taxa tendencial), a taxa nominal de juros seria igual a $4 \%$ (e, obviamente, a taxa real igual a $2 \%)$. Segundo Taylor, o comportamento de muitos bancos centrais, inclusive o FED, poderia ser explicado pela regra acima.

Nas condições acima descritas, a taxa nominal de juros se desviará da taxa adequada se $\dot{p}$ for diferente de $\ddot{p}^{*}$ e $y$ for diferente de zero. Desvios positivos de $Y$ em relação a $Y_{n}$ e de $\dot{p}$ em relação a $\ddot{p}^{*}$ levarão o banco central a aumentar a taxa de juro. Para esta função de reação, pode-se verificar à semelhança de Walsh que "uma reação de $i$, taxa nominal de juro maior do que um para um, assegura que a economia tenha um equilíbrio único, estacionário e de expectativas racionais" (Walsh, 2003, pp. 546-7).

Se considerarmos a regra acima podemos verificar que o estabelecimento de uma meta inflacionária $\ddot{p}^{\prime \prime}$, requer que as autoridades monetárias estabeleçam uma taxa de juro real igual à taxa de juro real de equilíbrio de longo prazo. Consideremos a regra dada por 1.8 acima, substituindo-se $i$ por $i_{r}+\dot{p}$ e supondo-se que no longo prazo $Y$ tende a ser igual a $Y_{n}$, teremos

$$
\begin{aligned}
& i_{r}+\dot{p}=i_{r}^{*}+p+h_{1}\left(\dot{p}-\dot{p}^{*}\right) ; \text { e em conseqüência } \\
& \dot{p}=\dot{p}+\frac{i_{r}-i_{r}}{h_{1}}
\end{aligned}
$$

o que significa que se o Banco Central estabelecer $\dot{p}=\ddot{p}^{*}$ deverá chegar uma taxa de juro real $i_{r}$ igual a $i_{r}^{*}$, ou seja, dada a inflação meta, deverá se estabelecer uma taxa de juro nominal tal que $i_{r}=i_{r}^{*}$.

\section{UMA AVALIAÇÃO CRÍTICA DO MODELO CONVENCIONAL DE METAS}

A introdução das metas inflacionárias como base da política monetária foi muito bem acolhida tanto pelos especialistas em bancos centrais, como também pelos economistas chamados de modo geral de "keynesianos". Estes viram aí um abandono dos mecanismos de transmissão monetaristas; para estes, um aumento da oferta monetária real, dada a baixa substitubilidade do dinheiro por outros ativos financeiros, levaria a um imediato aumento na demanda por ativos reais e à inflação, dada a suposição de pleno emprego automático. De outro lado, para os keynesianos, um aumento da quantidade de dinheiro ao elevar a demanda por ativos financeiros provocaria um aumento no preço destes e uma redução da taxa de juro levando a um aumento na demanda real e na renda, especialmente por não admitirem o pleno emprego de forma automática. De acordo com Keynes (1986), tal se daria a partir de três elementos:

“a) a função de preferência pela liquidez que nos diz em quanto a taxa de 
juro terá de cair a fim de o novo dinheiro ser absorvido pelos demandantes de liquidez;

b) a função de eficiência marginal do capital que nos diz em quanto uma queda na taxa de juro aumentará o investimento;

c) o multiplicador do investimento que nos diz em quanto um dado aumento no investimento aumentará a demanda como um todo" (p.298).

Não há dúvida de que a introdução da função IS nos novos modelos retoma o mecanismo de transmissão keynesiano; porém, ao se introduzir os conceitos de taxa de juro de equilíbrio de longo prazo (correspondente à taxa natural de Wicksell) e a hipótese de uma renda de equilíbrio de pleno emprego, ou o NAIRU realizando-se sempre, mediante a dinâmica ao longo $D A$, faz com que a política monetária a longo prazo, como observado, seja considerada absolutamente neutra, podendo afetar apenas os valores da taxa de juro nominal e da inflação, mantendo-se constantes a renda de pleno emprego e a taxa de juro real de equilíbrio.

A análise empírica tem mostrado que esta hipótese tem grandes limitações. Inicialmente, observa-se grande imprecisão na sua medida. De acordo com Staiger, Stock e Watson (1997), a estimativa do NAIRU para a economia americana, para o ano de 1994, dependendo da especificação do modelo, para um intervalo de confiança de $95 \%$, teve sua menor variação entre 4,8 e 6,6 pontos percentuais. Para esses autores, o NAIRU não é importante no processo de previsão da inflação; para eles, "previsões de inflação baseadas no desvio do desemprego, em relação ao NAIRU, são similares, quer se admita que o NAIRU assuma os valores de $4,5 \% 5,5 \%$ ou $6,5 \%$. As dificuldades para a estimação do NAIRU e seu papel limitado na previsão da inflação estão naturalmente inter-relacionados; pois se o NAIRU desempenhasse um papel mais importante em tal previsão, então seu valor poderia ser determinado com uma maior precisão dos dados [...] Uma conclusão extrema a se obter de tais resultados é a de que o NAIRU não existe. Tal argumento poderia ser baseado na hipótese que o NAIRU tem se deslocado ou então na existência de um intervalo de confiança muito amplo para sua estimativa" (p. 46). Ainda observam que não se pode depreender disso que não haja uma relação inversa entre taxa de desemprego e inflação. O que se observa, mesmo a longo prazo, é um trade-off entre a taxa de desemprego e inflação, como na curva de Philips tradicional.

Assim, para alguns propósitos como estimar o quanto a inflação aumentará para uma queda de $1 \%$ no desemprego, tal trade-off é relevante, o mesmo não podendo ser dito do NAIRU. "Para efeitos de política econômica positiva, basta saber que há uma regularidade empírica, embora vaga, entre taxa de desemprego e a taxa de inflação" (p. 47). De acordo com aqueles autores, utilizando-se o índice de preços ao consumidor o efeito predito de uma queda no desemprego de 5,5 para 4,5 pontos, relativos a uma base constante de 5,5\% de desemprego, é um aumento na taxa de inflação de 0,9 ponto percentual para o primeiro ano e um aumento de 1,5 ponto percentual acumulado para os primeiros dois anos. A 
inclinação desta relação de Philips foi estimada de maneira bastante precisa (o $t$ estatístico da soma dos coeficientes, com base no desemprego do ano anterior foi de $4,1 \%)$.

Quais as implicações de tais constatações? A primeira é a possibilidade de se estabelecer uma relação entre inflação e desemprego diferente da estabelecida no modelo convencional de regras, ou seja, pode-se aceitar que a taxa de inflação apresente um trade-off suave em relação às variações das taxas de desemprego, dentro de uma razoável amplitude destas, e que terá uma aceleração maior quando ultrapassa o limite máximo desta amplitude como na curva de Philips tradicional.*

Outra implicação decorrente desta é que variações na renda da economia não estão sujeitas a um limite dado por um valor pontual, $Y_{n}$, mas que ocorrerão de forma definitiva, e não temporária, mesmo que isto envolva uma elevação da taxa inflacionária. Decorre disto, que a política monetária, ou mesmo fiscal, não são neutras a longo prazo, uma vez que provoquem uma elevação na Demanda Agregada $(D A)$ e em conseqüência na renda. A possibilidade da elevação da renda potencial mediante a elevação da $D A$, e portanto da endogeneização da renda potencial, foi verificada empiricamente no trabalho de Leon Ledesma e Thirlwall (2002). Estes autores consideraram o comportamento de 15 países do OCDE no pós-guerra e mostraram inicialmente que a taxa de crescimento necessária para manter o nível de desemprego constante se eleva nos períodos de auge econômico e cai nas recessões, pois verificaram que o crescimento da força de trabalho e da produtividade são elásticas em relação ao crescimento da demanda e do produto. Tal foi confirmado usando-se testes de causalidade (teste de Granger) entre crescimento de "inputs" e "outputs", de tal modo que as teorias ortodoxas do crescimento e as "novas" teorias do crescimento, segundo as quais é o crescimento dos "inputs" que causa de maneira unidirecional o crescimento da renda, não encontram nenhuma confirmação empírica.

Tais resultados justificam também que, para efeito de determinação de uma taxa de juro de longo prazo, a equação (1.5) deixa de ter um valor único, mas pode-se alterar em função de variações da Demanda Agregada. Em tal contexto a taxa de juro de longo prazo não pode ser considerada legitimamente natural, na acepção de Wicksel, e deixa, portanto, de ser um parâmetro, tal como aparece na formulação algébrica da regra de Taylor (equação 1.8).

Assim, mesmo um wickselliano como Morishima (1984) admite que "a taxa natural de juro não é alguma coisa que possa ser medida e anunciada publica-

\footnotetext{
* Constatação semelhante encontra-se em Blinder (1997), para quem sucessivos estudos econométricos, nos anos 80, concluíram que a curva de Philips empírica encontrava-se viva e bem, desde que se considerassem os choques de oferta pelo menos nos Estados Unidos. O mesmo foi constatado por Gordon (1987) para a economia européia.
} 
mente, o melhor que se pode fazer é identificar se a taxa de juro nominal corrente é maior ou menor que a taxa natural, pela consideração de como as forças de mercado estão operando em um dado momento" (p. 271). Allan Blinder (1999) tem opinião análoga à de Morishima. Identificando a taxa natural com a taxa neutra de juro, observa que tal taxa "é difícil de calcular e impossível conhecê-la com precisão. Deve ser pensada de forma mais útil, antes como um conceito do que um número, como uma forma de se pensar sobre a política monetária e não como base para uma regra mecânica" (p. 33); Blinder ainda apresenta uma possibilidade de o banco central calcular tal taxa, porem tal cálculo pode levar "apenas a um intervalo e não a um ponto preciso" (p. 35).

Finalmente, outra hipótese questionável do modelo de metas, como enunciado por Taylor, é o processo de ajustamento da economia ao pleno emprego, toda vez que ocorra um choque de oferta favorável ou desfavorável alterando $Y$ $n$ para mais ou para menos. Em tais situações a $D A$ se deslocaria para cima ou para baixo de modo a determinar um equilíbrio de longo prazo correspondente ao novo nível de $Y$ n.

É o que supõe Clarida et al. (1996) ao afirmar que "uma elevação permanente na produtividade aumenta a renda potencial, mas aumenta também a demanda de maneira a compensar perfeitamente aquele aumento devido ao efeito da renda permanente" (p. 1675).

Um refinamento de tal proposição seria a de que ao aumentar a renda potencial da economia, mantida constante a oferta monetária, haveria uma queda da inflação e uma elevação do valor dos encaixes monetários, expandindo a riqueza das famílias e, portanto, aumentando a demanda de mercadorias e de títulos, elevando o preço destes e em conseqüência reduzindo a taxa de juro real e aumentando o investimento e a demanda agregada. Alguns problemas surgem, no entanto, quando se considera tal mecanismo. Talvez o mais significativo conforme assinala Palacio-Veras (2005, pp. 760-1), seja o de que excluindo-se os títulos governamentais nas mãos do setor privado, a riqueza financeira deste é aproximadamente igual à base monetária, sendo que esta representa uma proporção reduzida da oferta monetária nas economias modernas. Assim. a deflação necessária para gerar o aumento em $D A$ seria simplesmente catastrófica. $\mathrm{O}$ conjunto desses argumentos enfraquece a interpretação teórica sobre a qual se baseia a política atual de metas inflacionárias, o que justifica que se pense em um modelo alternativo de inflação e política monetária, como o que se propõe a seguir.

\section{UM MODELO ALTERNATIVO}

A proposta de uma alternativa à política de metas inflacionárias decorre das críticas que foram anteriormente apresentadas. Para tanto, inicialmente, se con- 
siderará que a renda potencial não é independente da demanda agregada. No modelo convencional esta é determinada exogenamente e depende basicamente do progresso técnico e do crescimento secular da força de trabalho. Define-se uma renda potencial única (correspondente ao NAIRU), que não se altera a curto e médio prazo, enquanto a economia se ajusta a esse valor, mediante modificação ao longo de uma determinada demanda agregada. Romer (2000) justifica tal hipótese, ao afirmar que está seguindo a prática usual de se negligenciar o fato de que a taxa natural do produto está crescendo com o tempo (p. 160). Por razões dadas anteriormente tal prática será descartada.

Além disso, na proposta alternativa, não se considerará a taxa natural de juro como um parâmetro dado em um certo momento, na determinação de meta inflacionária, como acontece na regra de Taylor, pois de acordo com a observação de Blinder (1999), na melhor das hipóteses o "cálculo dessa taxa (para um período de tempo) levará a um intervalo e não a um ponto” (p. 35).

Consideremos assim uma economia fechada, produzindo apenas um bem que pode ser usado para consumo ou para investimento, sem setor governo e inicialmente sem considerar setor monetário. Tal economia tem uma equação do produto dada por $Y=C L+g K$, sendo $Y$ o produto, $L$ o valor da mão-de-obra empregada, $\mathrm{C}$ o consumo por unidade de mão-de-obra, $K$ o valor do estoque de capital e g a taxa de crescimento do capital. Dividindo-se a equação acima por $Y$, supondo-se que a razão emprego/produto é um coeficiente tecnológico fixo, dado por $a_{o}$, obtém-se

$$
1=C a_{0}+g \frac{K}{Y}
$$

De acordo com (3.1), um maior consumo por trabalhador está associado com uma redução da taxa de crescimento da relação capital/produto,em termos reais, $\frac{K}{Y}$. Definindo-se $a_{1}$, como a relação capital produto podemos ter duas situações: a igualdade $\left(\frac{K}{Y}=a_{1}\right)$ significa que a economia encontra-se em situação de pleno emprego, ao passo que a desigualdade

$$
\left(\frac{K}{Y}\right) \leq a 1
$$

corresponde a uma situação em que a economia encontra-se operando com excesso da capacidade, isto é, não está gerando a renda de pleno emprego.

Além da equação do produto, pode-se definir uma equação de preços por

$$
P=W a_{o}+r\left(\frac{K_{n}}{Y}\right)
$$

onde $P$ é o preço; $W$ o salário nominal, $r$ a taxa de lucro e $K_{\mathrm{n}} \mathrm{o}$ valor nominal do capital. Dividindo por $P$, temos 


$$
1=\left(\frac{W}{P}\right) a_{o}+r\left(\frac{K}{Y}\right)
$$

onde $K$ seria o capital representado em termos reais.

Tal equação indica que a taxa de salário varia inversamente com a taxa de lucro dado o valor de $\left(\frac{\kappa}{r}=a\right)$. O mesmo não será verdadeiro se a economia estiver com uma utilização baixa de sua capacidade produtiva, isto é, com $\frac{\kappa}{\bar{r}} \geq a$, o que é bastante realista se considerarmos que as firmas em economias capitalistas não utilizam, em geral, plenamente seu capital.

O sistema formado por (3.1), (3.2) e (3.3) estabelece um conjunto de cinco variáveis (C, g, $\frac{W}{P}$, r e $\left.\frac{K}{Y}\right)$; duas equações (3.1) e (3.3) e uma desigualdade (3.2). Daí a necessidade de mais três equações para resolver o sistema. Pode-se obter tais equações supondo-se que os assalariados não poupam e os capitalistas não consomem, o que significa que a taxa de crescimento da economia (g) é igual à taxa de retorno $(r)$ sobre o capital. Um sistema mais realista não alterará as conclusões a que se chegará com tal simplificação. Obtemos assim

$$
\mathrm{g}=r
$$

ou simplesmente $\frac{W}{P}=c$. Uma destas duas equações é redundante, sendo dadas as hipóteses relativas ao consumo dos assalariados e dos capitalistas, isto é, as equações (3.1) e (3.3).

Pode-se supor também que

$$
\left(\frac{W}{P}\right)=\left(\frac{W}{P}\right)^{*}
$$

sendo $\left(\frac{W}{P}\right)^{\circ}$ o salário convencional determinado exogenamente pelas condições do mercado de trabalho.

Estas suposições vão requerer mais duas equações para fechar o modelo. Uma delas é dada pela determinação de preços pelas firmas, que baseada em Kalecki estabelece que as firmas aplicarão um mark-up (z) sobre os custos diretos de produção tal que

$$
P=W a_{o}+z W a_{o}=(1+z) W a_{o}
$$

do que decorre

$$
\left(\frac{W a_{o}}{P}\right)=\frac{1}{\left[(1+z) a_{o}\right]}
$$

onde $z$, o mark-up, é determinado pelas condições da concorrência entre as firmas e outros fatores relacionados com a organização industrial; e a equação restante será dada pela taxa de crescimento das empresas, que dependerá, segun- 
do Steindl, da taxa de lucro e do grau de utilização da capacidade instalada, $\frac{Y}{K}$, ou seja

$$
g=g\left(r, \frac{Y}{K}\right)
$$

sendo que $\mathrm{g}$ responde positivamente aos dois argumentos.

De outro lado, substituindo-se (3.7) em (3.3), obtém-se $\left(\frac{Y}{K}\right)=\frac{r(1+z)}{z}$, que combinada com estabelece

$$
g=g\left[r, r \frac{(1+z)}{z}\right]
$$

a qual estabelece uma relação positiva entre g e $r$ sendo dado $z$.

Seria importante mostrar-se, agora, que elevações no salário real podem determinar taxas de crescimento e taxas de lucro mais elevadas dada a hipótese $\frac{K}{Y} \geq a_{1}$.

Os gráficos abaixo, elaborados por Dutt (1987), sugerem que a taxa de acumulação desejada poderia ser aumentada com um aumento no salário real, o que corresponderia a uma redução do valor de z.

\section{Gráfico 2}

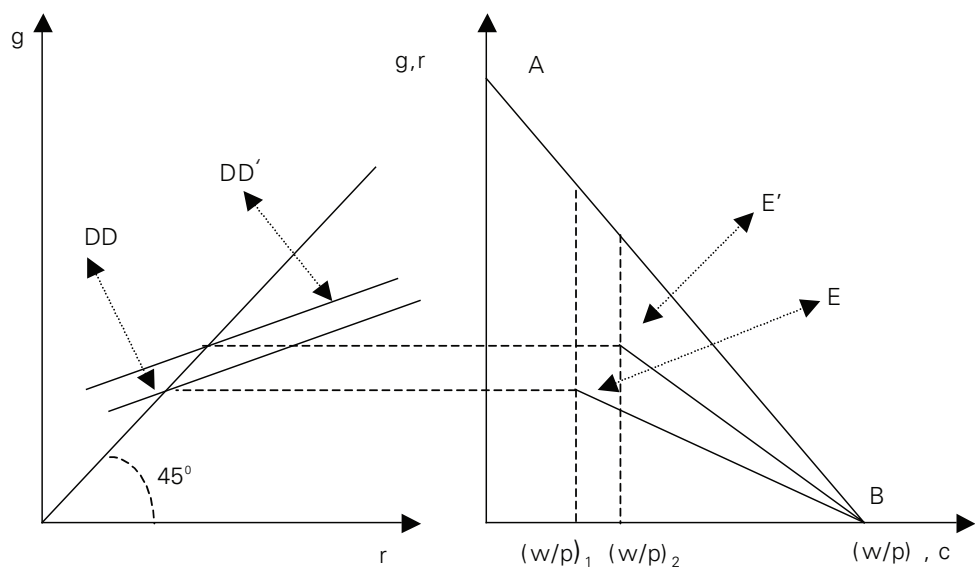

A linha $A B$ indica as condições de funcionamento da economia à plena capacidade, $\operatorname{com} \frac{K}{Y}=a$, de tal maneira que uma elevação de $(w / p)$, ou $c$, levaria a uma redução de $g$ ou $r$. Consideremos, agora, a possibilidade de $\frac{K}{Y}=a_{0} \geq a_{1}$. Nesta situação podemos estabelecer um equilíbrio dentro do espaço limitado pela linha $A B$ e a origem, por hipótese, o ponto $E$, que corresponde a um salário real $(w / p)_{1}$, associado a uma linha taxa de lucro/salário real (ou taxa de crescimento/consumo), 
que tendo início em $E$ terminará em $B$, cuja inclinação, como podemos ver a partir da equação (3.3), será $-a_{0}(Y / K)$. Podemos ver que, neste caso, o equilíbrio será dado pela intersecção de $D D$ com a reta de $45^{\circ}$.

Consideremos, agora, a hipótese de que uma queda, mesmo que temporária, em $z$, leve a um aumento do salário real, de $(w / p)_{1}$ para $(w / p)_{2}$. Tal mudança levaria a um aumento na taxa de lucro $r$ e em $g$. Neste caso, uma redistribuição de renda, mesmo que temporária, dos capitalistas, que não consomem (ou consomem muito pouco), para os trabalhadores, que consomem praticamente tudo o que recebem na forma de salários, aumentaria a demanda agregada, levando os empresários, num primeiro momento, a utilizar mais de sua capacidade instalada para atender a esse aumento na demanda, e, num segundo momento, a investir mais, provocando um aumento na taxa de crescimento da economia.

É possível incorporar-se o efeito da variação do salário real sobre a taxa de crescimento, substituindo-se em (3.9) o valor de z, tal como aparece na equação de preço.

A partir desta equação, como representada abaixo,

$P=W a_{o}+z W a_{o}=(1+z) W a_{o}$, podemos obter imediatamente um valor para z, dado pela expressão

$$
z=\left(\frac{P}{W a_{0}}\right)-1
$$

que, substituída em (3.9), permite estabelecer

$$
g=g\left(r,\left(\frac{r P}{P-W a_{a}}\right)\right)
$$

a qual indica que $g$ vai variar diretamente com $W$, mantido $P$ constante. Assim, uma elevação em $W$ está representada no Gráfico 2, por um deslocamento para cima de $D D$ para $D D^{\prime}$ implicando valores maiores para $g$ e $r$. Pode-se mostrar também que $g$ se elevará a taxas crescentes dada uma variação de $W^{*}$. Tal constatação será relevante para a análise da inflação que será feita adiante.

\section{O processo inflacionário e a política monetária}

A análise do processo inflacionário vai pressupor que as variações nas taxas de crescimento estão relacionadas com variações nas taxas salariais e, portanto, com os custos de produção e os preços. Tal modelo vai considerar que, dada uma taxa de "mark-up", tanto os empresários como os assalariados determinarão metas salariais próprias: os assalariados visando aumentar o salário real e sua participação na renda e os empresários visando aumentar a sua lucratividade. A do-

\footnotetext{
* A derivada de $\mathbf{g}$ em w é positiva.
} 
minação de uma tendência ou outra determinará valores diferentes para a demanda e o crescimento.

Definindo-se w como salário real pode-se explicitar a inflação salarial $\dot{w}$ como uma função da diferença entre a meta salarial $\left(w_{w}\right)$ e o salário efetivamente pago $(w)$

$$
\dot{w}=H\left(w_{w}-w\right)
$$

De forma análoga, a inflação de preços será definida por uma função J

$$
\dot{p}=J\left(w-w_{f}\right)
$$

sendo $w_{f}$ o salário que os empresários consideram adequado pagar, tendo em vista uma lucratividade desejada. Supondo-se que as expectativas inflacionárias tanto dos assalariados quanto dos empresários sejam adequadas, igualandose $\dot{p}$ e $\dot{w}$, dados em (3.11) e (3.12), existirá um valor de equilíbrio de $w^{*}$

$$
w^{*}=\frac{H w_{w}+J w_{f}}{J+H}
$$

A equação (3.13), indica que a taxa de variação do salário de equilíbrio $w^{*}$ depende da média ponderada das metas de salários determinadas pelos assalariados e pelas empresas, os pesos dependendo dos coeficientes de ajustamentos de salários e preços, $\mathrm{H}$ e J.

Para se identificar o efeito da política monetária no modelo, será necessário incorporar à análise o efeito da taxa de juro real $i_{r}$, e não apenas a taxa de juro nominal, devido ao efeito da inflação. Se supusermos agora que os empresários podem estabelecer valores para seu mark-up ("target return pricing”) definimos

$$
z=z_{0}+z_{r}
$$

Tal relação pode ser justificada porque a elevação de $i_{r}$ aumentará o custo de $i_{r}$ oportunidade do investimento.

No caso em que as firmas não podem estabelecer seu mark-up, o efeito do aumento de $i_{r}$ pode ser interpretado em termos de um "salário real meta" estabelecido pelas firmas (targeting real wage). Considerando-se a possibilidade de o Banco Central estabelecer a taxa de juro real pelo controle de i (taxa de juro nominal), pois $i_{r}=i-\dot{p}$, o salário real adequado para as firmas será dado por

$$
w_{f}=w_{f o}-\beta\left(i_{r}\right)
$$

sendo que o coeficiente $\beta$, indica o efeito que uma variação da taxa de juro real terá sobre o salário meta estabelecido pelas firmas. Neste caso, o salário de equilíbrio será menor do que o definido em (3.13), isto é, será dado por

${ }^{2}$ Apresentação similar à apresentada por Dutt (1987). Mencionada em Lavoie (1992). 


$$
w^{*}=\frac{H w_{w}+J\left(w_{f 0}-\beta\left(i_{r}\right)\right)}{H+J}
$$

Outro elemento desta análise é a determinação da meta salarial dos trabalhadores, $w_{w}$. A hipótese a ser considerada é uma curva de Philips de longo prazo, estabelecendo uma relação direta entre a taxa de crescimento e taxa inflacionária, com a modificação de que a taxa de salário desejada pelos assalariados é uma função da taxa de crescimento do emprego e não do nível de desemprego. Assim, se o emprego está crescendo, em função de um aumento de g, a demanda de mão-debra aumentará e o salário meta crescerá também, como estabelece (3.17)

$$
\begin{aligned}
& w_{w}=w_{w o}+H_{g} g \\
& \text { O gráfico abaixo representa tais relações: }
\end{aligned}
$$

\section{Gráfico 3}

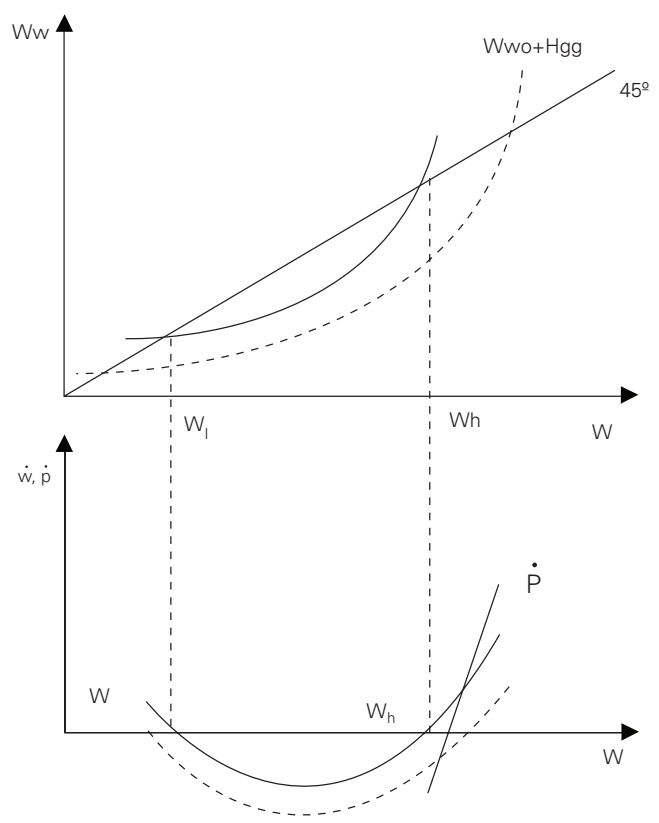

Na parte superior do Gráfico 3 (construído a partir de Lavoie, 1991, p. 408) está representada a equação (3.17), com uma linha com inclinação positiva crescente. A forma desta curva pode ser explicada pela equação (3.11), que indica que conforme $\mathrm{w}$ aumenta obtemos taxas de crescimento positivas e crescentes, $\mathrm{o}$ que decorre de a primeira e a segunda derivadas de g em relação a w serem positivas. Os pontos $w_{b}$ e $w_{1}$ indicam os valores de $\mathrm{w}$ em que $w_{w}=w$, pois correspondem à intersecção da função dada por (3.17), com uma linha de $45^{\circ}$.

$\mathrm{Na}$ parte inferior do gráfico está representada a função $\dot{w}$ como a diferença 
entre $H w_{\text {wo }}+H_{g} g$ e a linha de $45^{\circ}$. Nos valores entre $w_{1}$ e $w_{b}, \dot{w}$ tem os valores negativos; à direita de $w_{h}, \dot{w}$ passa a ter valores positivos. Assim, nos valores de $w$, correspondentes a $w_{1}$ e $w_{b}$, a inflação salarial será zero, e o salário será igual ao valor do salário reivindicado pelos trabalhadores, isto é, $w=$ $w_{w}$.

O Gráfico 3 ilustra, ainda, outras possibilidades, cujas implicações serão discutidas adiante. Uma elevação da taxa de juro que reflita uma redução do salário de equilíbrio, como mencionado anteriormente, tem como conseqüência reduzir o valor de g (conforme equação 3.10) deslocando as curvas representadas nas partes superior e inferior do gráfico, o que aparece através das linhas pontilhadas.

De outro lado, a determinação da taxa de inflação efetiva vai requerer que se considere o processo de decisão de preço das empresas. Para tanto, vamos supor inicialmente que tais decisões são independentes da taxa de crescimento da economia. Assim temos uma função $\dot{p}$ com inclinação positiva que pode ser representada na parte inferior do gráfico acima mencionado. De acordo com a equação 3.12, elevações em $\dot{w}$ terão como conseqüência uma elevação nos valores de $\dot{p}$.

Dadas as condições acima, pode-se analisar o efeito de algumas decisões de políticas monetárias. Consideremos uma situação em que as firmas têm controle sobre o seu mark-up (target return prices) e que as autoridades elevem a taxa de juro. Neste caso, de acordo com a equação (3.12), as empresas para manter sua lucratividade elevarão o seu mark-up para todos os níveis de g, como representado no gráfico 4a, abaixo, que é uma transposição do lado inferior direito do Gráfico 3. Tal significaria um deslocamento de $\dot{p}$ para $\dot{p}$ determinando uma taxa de crescimento e uma taxa de inflação menores. No caso alternativo de as empresas não terem controle sobre seu mark-up, de acordo com as equações (3.15) e (3.16) haveria um deslocamento da curva $w$ mantendo-se fixa a função $\dot{p}$, provocando resultados análogos ao do primeiro caso, isto é uma redução da taxa de inflação e da taxa de crescimento, tal como representado Gráfico 4b. Obviamente o contrário acontecerá para uma redução na taxa de juro real.

\section{Gráfico 4}
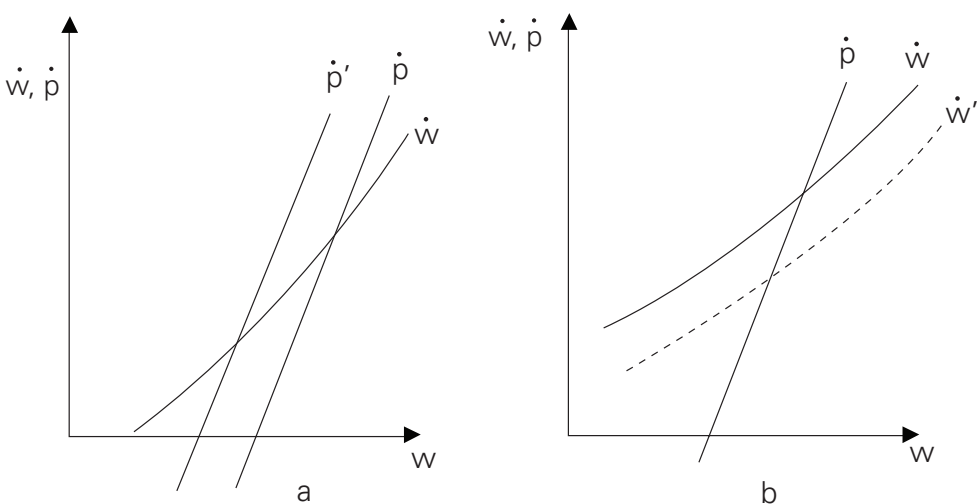

b 
Deve-se assinalar que a escolha do lado direito inferior do Gráfico 3, com ascendente, não é arbitrária, mas decorre da verificação empírica da relação direta entre a taxa de inflação e emprego ou alternativamente da relação inversa entre a taxa de inflação e taxa de desemprego, como indicada por uma curva de Philips convencional. Tais relações correspondem à equação (3.17), desde que nela se interprete que uma variação positiva em $w$ corresponda a um aumento do emprego ou a uma diminuição na taxa de desemprego. Tais relações, como discutidas na segunda seção, podem ser consideradas válidas empiricamente, tanto para o curto como para o longo prazo.

Assim como ilustrado em $4 \mathrm{a}$ e $4 \mathrm{~b}$, o trade off entre crescimento e inflação é permanente e não apenas válido no curto prazo. Entretanto, pode haver limites severos para a redução da taxa de juro real para estimular a produção. Segundo Smithin (1997), os interesses financeiros dificilmente tolerariam uma situação em que a taxa de juro real se tornasse negativa ou próxima de zero, como provou o desenvolvimento histórico do último quarto do século XX. De fato, podese assim aceitar que são considerações políticas, que em última instância previnem a adoção de uma política de taxas de crescimento elevadas no capitalismo contemporâneo, e não conceitos teóricos como o NAIRU. Este conceito, na verdade, constitui-se em uma racionalização técnica dessa situação" (p.402). Podese acrescentar a esse argumento que os surtos recentes de crescimento, como o da economia americana, tem sido interpretados pela teoria convencional não como o resultado de uma política explícita de crescimento do produto e do emprego, mas sim como bolhas especulativas ou não.

Deve-se observar ainda que a curva de Philips não resume toda a história da inflação. Existe um elemento de custos que depende da variação do salário real definido como base para a determinação dos lucros e da produtividade média. Um aumento ou diminuição do "gap" entre essas variáveis contribuirá positiva ou negativamente para a inflação.

\section{CONSIDERAÇÕES FINAIS}

O modelo proposto na terceira parte deste trabalho não supõe que exista uma única taxa de juro de equilíbrio de longo prazo como também não supõe que exista um valor determinado da renda potencial. Esta vai depender do comportamento da demanda agregada que pode ser determinada por decisões de política econômica. Ainda, de acordo com o modelo alternativo, haveria um tradeoff entre crescimento e inflação, mesmo no longo prazo, que seria decidido pelas autoridades monetárias, tomando-se como base a taxa de juro real.

Contrariamente à idéia de Milton Friedman de que uma redução da taxa de juro nominal levaria a uma aceleração inflacionária, como suposto na curva de Philips ampliada pelas expectativas — pois neste caso com a elevação da infla- 
ção se criariam expectativas de maiores reduções na taxa de juro real, gerando um movimento inflacionário cumulativo - no modelo alternativo a taxa de juro real desejada seria tornada pública o que neutralizaria as expectativas de juros reais mais baixos.

Assim, na medida em que um modelo de estática comparativa pode servir de guia para a política econômica, os resultados desta análise sugerem que um banco central que esteja preocupado também com a produção e o emprego e não apenas com o controle da inflação, isto é, abrisse parcialmente mão de inflação muito baixa em favor de uma taxa de crescimento mais elevada, deveria perseguir uma política de dinheiro barato, dados os limites acima mencionados, no sentido de estabilizar a taxa de juro real em um valor baixo ainda que positivo. Isto pode ser conseguido mediante um ajustamento das taxas de juro nominais para compensar alterações nas taxas de inflação esperada pelo público, cuja previsão dependeria de modelos estatísticos construídos pelos próprios bancos centrais. Tais decisões, se disseminadas amplamente, contribuiriam de outro lado para a formação, por parte dos agentes econômicos, de suas expectativas futuras em relação às taxas de juros reais, com os efeitos positivos para a previsibilidade do comportamento da economia, com todos os benefícios que isto pode trazer.

De modo geral, a principal conclusão a ser tirada do modelo alternativo é a de que, na medida em que fica aberta a opção para as autoridades monetárias, de escolherem menos desemprego e mais inflação ou vice-versa, a opção escolhida dever ser comunicada e justificada para a sociedade. A determinação do instrumento de política econômica e de sua intensidade, certamente, envolve incertezas significativas decorrentes da mutabilidade do ambiente macroeconômico, de tal modo que metas definidas de maneira rigorosa não podem substituir paradigmas flexíveis de gestão de risco que dependem do discernimento e de formulação criteriosa das autoridades monetárias, diante de conjunturas específicas. A idéia de se estabelecer metas precisas, definindo-se pontos percentuais e decimais destes, como ocorre com o nosso banco central, conflita com as observações acima, pois supõe a possibilidade de definir de maneira rigorosa alguns parâmetros para a quantificação dessas metas, ou seja, a taxa de juro de equilíbrio de longo prazo (taxa natural de juro) e a taxa natural de desemprego (NAIRU).

\section{REFERÊNCIAS BIBLIOGRÁFICAS}

BLANCHARD, O. "Macroeconomia”, tradução da $2^{a}$ edição americana, 2001, Editora Campus.

BLINDER, A. (1997) "The Fall and Rise of Keynesian Economics” Economic Recorder dezembro. Também publicado em “A Macroeconomic Reader”, editores Brian Snowdon e Howard Wane, Routledge.

BLINDER, A. (1999) Central Banking in the Theory and Practice. Cambridge, MA: The MIT Press. CLARIDA, R., GERTER, M. e GALLI, J. (2000) “Monetary Rules and Macroeconomic Stability, Evidence and Some Theory”, Quarterly Journal of Economics, 2000, 115, pp. 147-78. 
DUTT, A.K. (1987) “Alternative closures again: a comment on growth, distribution and inflation", Cambridge Journal of Economics, 11: 75-82.

FRIEDMAN, M. (1968) “The Role of Monetary Policy”, American Economic Review 58, março, pp. 1-17.

GORDON, Robert J. (1987) "Wage Gaps vs. Output gaps: Is there a common history for all Unemploy men of Europe”, National Bureau of Economic Research, Working Paper 2454, dezembro.

GREENSPAN, A. (2004) "Risk and Uncertainty in Monetary Policy" American Economic Review, maio 2004 , volume $94, \mathrm{n}^{\circ} 2$.

KEYNES, J.M. (1936) “The General Theory”. McMillan, p. 298.

LAVOIE, M. (1992) "Foundations of Post-Keynesian Economic analysis", Edward Elgor Publishing Company, England, 1992.

LEON LEDESMA, M.A. e THIRWALL, A.P. (2002) “The Endogeneity of the Natural Rate of Growth", Cambridge Journal of Economics, vol. 26, pp. 442-59.

MANKIW, N.G. (1998) Macroeconomia. Editora LTC, São Paulo.

MINELLA, A. Freitas, P.S.; Goldfajn, I; e Muinhos (2002) "Inflation Targenting in Brazil — Lessons and Challenge”, Working Paper 53, Banco Central do Brasil, nov. 2002.

MORISHIMA, M. (1984) “The Economics of Industrial Soviety”, Cambridge University Press, G. Bretain, 1984.

PALACIO-VERAS, A. (2005) “The Modern View of Macroeconomics: Some Critical Reflections". Cambridge Journal of Economics, 29, 747-767

ROMER, DAVID, (2001) "Keynesian Macroeconomics Without the LM Curve", Journal of Economic Perspectives, vol. 14 n $^{\circ}$ 2, pp. 149-169.

SMITHIN, J. (1997) "An Alternative Monetary Model of Inflation and Growth, Review of Political Economy 1997 Volume 9, n ${ }^{\circ}$ pp. 395-409.

STAIGER, D., STOCK, J.H e WATSON, M.(1997) “The NAIRU, Unemployment and Monetary Policy”, Journal of Economic Perspectives, 1997 vol. II, nº 1, Winter, pp. 233-49.

TAYLOR, J. (1993) "Discretion versus Policy Rules in Practice, Carnegie, Rochester, Series on Public Policy 39, pp. 195-214.

TAYLOR, J. (1998) “Monetary Guidelines for employment and Inflation Stability" em "Inflation Unemployment and Monetary Policy”, editores Robert Solow e John Taylor, Cambridge Massachussets, MIT Press.

TAYLOR, J. (2000) “Teaching Modern Macroeconomics at the Principle Level”, American Economic Review, maio 90,(2) 200, pp. 90-4.

WALSH, C.E. (2003) “Monetary Theory and Policy, $2^{\text {nd }}$ edition, The MIT Press, Cambridge, Mass, Rondom, 2003. 